\title{
Cough hypersensitivity syndrome: clinical measurement is the key to progress
}

\begin{abstract}
To the Editor:
The recent European Respiratory Society (ERS) Task Force Report on chronic cough [1] should be commended for drawing attention to a neglected area of respiratory medicine and discussing controversies surrounding the approach to this common clinical problem. Cough hypersensitivity syndrome is proposed as a new shorthand to refer to patient-reported excessive coughing that otherwise evades clinical explanation [1]. As discussed in the report, cough hypersensitivity syndrome may or may not be associated with other causes of chronic cough, and its peripheral and central mechanisms are only partly understood [1].
\end{abstract}

The comparison of cough hypersensitivity syndrome with chronic obstructive pulmonary disease (COPD) as an umbrella term [1] is useful in recognising the existence of distinct cough phenotypes. These might be described according to underlying pathology, for example excessive coughing associated with: airway inflammation (asthma or bronchitis); infection (respiratory tract infection, pneumonia or tuberculosis); anatomic airway distortion (lung cancer or fibrosis); mechanical irritation (acid reflux or post-nasal drip); and cough in the absence of known pathology. The analogy ends there. While COPD has clear diagnostic criteria involving objective measurement this is not the case for cough hypersensitivity syndrome.

Cough, as a clinical problem, and as currently discussed in guidelines and consensus statements, is a symptom. This is distinct from coughing reported by an observer, cough measured objectively by acoustic monitoring, or cough induced experimentally by an inhaled irritant. Indeed, not only does patient-reported chronic cough correlate poorly with cough reflex sensitivity to inhaled capsaicin or citric acid (as acknowledged by the task force [1]), it also correlates only moderately with objectively measured cough frequency [2]. Patient-reported cough improves with placebo (as in randomised controlled trials of proton pump inhibitors [1]) and behavioural training [3]. It can also improve spontaneously, despite a long duration and failed trials of treatment [4]. Explanation of these facts is currently limited.

Basic questions about cough remain unanswered. There are few descriptions of how much coughing is "normal" (and how much should be regarded as pathological). Improved understanding of cough will only come about through measurement and objective description of clinical and physiological phenomena. The objective measurement of cough frequency is of prime importance. This should be carried out in a large

TABLE 1 Clinical tools for measurement of cough

Variable

Tool

Adaptable to routine

References practice?

\section{Cough symptoms}

Subjective cough severity

Cough-related quality of life

Objective description of coughing

Temporal cough patterns ${ }^{\#}$

Force/intensity of coughs

Sensitivity to inhaled irritants

Cough mechanism

Airway inflammation

Neuronal mechanisms

Central pathways

Genetic predisposition
Visual analogue scale

Leicester cough questionnaire, cough-specific quality of life questionnaire

Ambulatory cough monitoring

Airflow measurements, electromyography, oesophageal pressure monitoring

Inhalational cough challenge

BAL sample analysis, induced sputum cell counts, exhaled nitric oxide fraction, exhaled volatile organic compounds

Animal models, human surgical and autopsy specimens

Functional magnetic resonance imaging

Genotyping for single nucleotide polymorphisms of potential genes including TRPV1

$\begin{array}{cc}\text { Yes } & {[6]} \\ \text { Yes } & {[6]} \\ & \\ \text { Yes } & {[6,7]} \\ \text { Possibly } & {[7]} \\ \text { Possibly } & {[6]} \\ \text { Yes } & {[1,9]} \\ \text { No } & {[6]} \\ \text { Possibly } & {[10]} \\ \text { Possibly } & {[8,10]}\end{array}$

BAL: bronchoalveolar lavage. " : daily cough frequency, diurnal cough pattern and degree of clustering of coughs into bouts. 
range of patients and respiratory conditions, as well as in healthy controls, and repeated over time, during the natural history of disease and in response to treatments. This will be made possible by agreement on how to count coughs, and the further development and increased availability of automated cough monitoring [5-7]. Objective cough counts should then be related to clinical and genetic variables [8], measures of respiratory tract inflammation (possibly including profiles of exhaled volatile organic compounds (VOCs) [9]) and neuronal and central factors [1, 10], and compared with subjective concerns and symptoms (table 1) [6,7]. In this way cough phenotypes will be much more rigidly defined in terms of symptoms, coughing behaviour and mechanisms.

Many of these measurements can be employed in routine clinical practice, at least in specialist clinics, although the available tools are currently in varying stages of development. For example, automated or semi-automated cough monitors are still being refined and are currently only used in research groups [7]. An ambulatory method of measuring cough intensity is required [7]. Available standard inhalation cough challenge tests do not currently differentiate health from disease $[1,6]$. Analysis of exhaled VOCs is still in its infancy [9], the cost of functional magnetic resonance imaging and genotyping may be a limiting factor, and the genetic basis of variations in cough perception and frequency has yet to be elucidated [1].

A previous ERS task force addressed the assessment of cough 8 years ago [6]. Unfortunately, there have been few advances in the tools available to measure cough since then. This is a priority for cough research. Measurement will not only allow cough hypersensitivity syndrome and other phenomena to be characterised with greater precision, it is the key to progress in this field.

@ERSpublications

We call for wider measurement of clinical variables in cough in order to define phenotypes and advance cough research http://ow.ly/JXnIz

Richard D. Turner and Graham H. Bothamley

Dept of Respiratory Medicine, Homerton University Hospital NHS Foundation Trust, London, UK.

Correspondence: Richard D. Turner, Dept of Respiratory Medicine, Homerton University Hospital NHS Foundation Trust, London, E9 6SR, UK. E-mail: richard.turner@homerton.nhs.uk

Received: Nov 182014 | Accepted after revision: Dec 132014

Conflict of interest: None declared.

\section{References}

1 Morice AH, Millqvist E, Belvisi MG, et al. Expert opinion on the cough hypersensitivity syndrome in respiratory medicine. Eur Respir J 2014; 44: 1132-1148.

2 Decalmer SC, Webster D, Kelsall AA, et al. Chronic cough: how do cough reflex sensitivity and subjective assessments correlate with objective cough counts during ambulatory monitoring? Thorax 2007; 62: 329-334.

3 Chamberlain S, Garrod R, Birring SS. Cough suppression therapy: does it work? Pulm Pharmacol Ther 2013; 26: 524-527.

4 Yousaf N, Montinero W, Birring SS, et al. The long term outcome of patients with unexplained chronic cough. Respir Med 2013; 107: 408-412.

5 Turner RD, Bothamley GH. How to count coughs? Counting by ear, the effect of visual data and the evaluation of an automated cough monitor. Respir Med 2014; 108: 1808-1815.

6 Morice AH, Fontana GA, Belvisi MG, et al. ERS guidelines on the assessment of cough. Eur Respir J 2007; 29: $1256-1276$.

7 Spinou A, Birring SS. An update on measurement and monitoring of cough: what are the important study endpoints? J Thorac Dis 2014; 6: Suppl. 7, S728-734.

8 Smit LAM, Kogevinas M, Antó JM, et al. Transient receptor potential genes, smoking, occupational exposures and cough in adults. Respir Res 2012; 13: 26.

9 van de Kant KDG, van der Sande LJTM, Jöbsis Q, et al. Clinical use of exhaled volatile organic compounds in pulmonary diseases: a systematic review. Respir Res 2012; 13: 117.

10 Morice AH, Jakes AD, Faruqi S, et al. A worldwide survey of chronic cough: a manifestation of enhanced somatosensory response. Eur Respir J 2014; 44: 1149-1155. 